\title{
HOBSON'S FORMULA FOR DUNKL OPERATORS AND ITS APPLICATIONS
}

\author{
NOBUKAZU SHIMENO
}

\begin{abstract}
We generalize classical Hobson's formula concerning partial derivatives of radial functions on a Euclidean space to a formula in the Dunkl analysis. As applications we give new simple proofs of known results involving Maxwell's representation of harmonic polynomials, Bochner-Hecke identity, Pizzetti formula for spherical mean, and Rodrigues formula for Hermite polynomials.
\end{abstract}

\section{INTRODUCTION}

There exists a nice explicit formula due to Hobson [14, 15] to calculate the action of a constant coefficient linear differential operator on radial functions on a Euclidean space, though it is not very well known. There are several applications of Hobson's formula such as Clebsch projection and Maxwell's representation of harmonic functions, Bochner-Hecke identity, and formulae for Hermite polynomials ([14, 15, 25, 26]). Nomura [20] gives a new simple proof of Hobson's formula by using the Euler operator.

In this paper we prove an analogue of Hobson's formula for the Dunkl operators (Theorem 11) by using a method similar to that of [20. The Dunkl operators are differentialdifference operator on a Euclidean space $\mathbb{R}^{d}$ associated with a finite reflection group $G$, which are deformations of directional derivatives. For $G=\mathbb{Z}_{2}^{d}$, our formula is previously given by Volkmer [30]. Moreover, our formula contains the original Hobson's formula as a special case. The formula for general $G$ seems to be a new result.

We give some applications of Hobson's formula for the Dunkl operators analogous to those for the Euclidean case mentioned above. Though consequences of Hobson's formula presented in this paper are all known results, they provide simpler alternative proofs or other viewpoints in the Dunkl analysis. We believe that Hobson's formula is worth more attention in Dunkl analysis as well as in Euclidean Fourier analysis.

\section{Preliminary Results on Dunkl analysis}

In this section we give notation, definitions, and some of known results on Dunkl analysis. We refer [9, 23, 29] for details.

Date: April 5, 2018.

2000 Mathematics Subject Classification. Primary 33C52, Secondary 33C45, 42B10.

Key words and phrases. Hobson's formula; Dunkl operators; Bochner identity; Pizzetti's formula. 
Throughout this paper let $i$ denote the imaginary unit $i=\sqrt{-1}$. Let $d$ be a positive integer. Let $\langle$,$\rangle be the standard inner product on \mathbb{R}^{d}$ and put $\|x\|=\langle x, x\rangle^{1 / 2}$ for $x \in \mathbb{R}^{d}$. Let $R \subset \mathbb{R}^{d}$ be a reduced root system, which is not necessarily crystallographic. For $\alpha \in R$, we write $r_{\alpha}$ for the reflection with respect to the hyperplane $\alpha^{\perp}$. Let $G$ denote the finite reflection group generated by $\left\{r_{\alpha}: \alpha \in R\right\}$. We fix a positive system $R_{+} \subset R$.

Let $\mathscr{P}=\mathscr{P}\left(\mathbb{R}^{d}\right)$ denote the space of polynomials on $\mathbb{R}^{d}$. For a non-negative integer $m$, let $\mathscr{P}_{m}$ denote the space of polynomials on $\mathbb{R}^{d}$ that are homogeneous of degree $m$.

Let $\kappa: R \rightarrow \mathbb{R}_{\geq 0}, \alpha \mapsto \kappa_{\alpha}$ be a $G$-invariant function on $R$. We call $\kappa$ a (non-negative) multiplicity function. We define

$$
\gamma_{\kappa}=\sum_{\alpha \in R_{+}} \kappa_{\alpha}, \quad \lambda_{\kappa}=\gamma_{\kappa}+(d-2) / 2 .
$$

For $\xi \in \mathbb{R}^{d}$, let $\partial_{\xi}=\langle\xi, \nabla\rangle$ denote the directional derivative corresponding to $\xi$ and define the Dunkl operator $\mathcal{D}_{\xi}=\mathcal{D}_{\xi}(\kappa)$ by

$$
\mathcal{D}_{\xi} f(x)=\partial_{\xi} f(x)+\sum_{\alpha \in R_{+}} \kappa_{\alpha}\langle\alpha, \xi\rangle \frac{f(x)-f\left(r_{\alpha} x\right)}{\langle\alpha, x\rangle} .
$$

The Dunkl operators satisfy $\left[\mathcal{D}_{\xi}, \mathcal{D}_{\eta}\right]=0$ for all $\xi, \eta \in \mathbb{R}^{d}([7])$. Here $[A, B]:=A B-B A$ for operators $A, B$. Let $\left\{e_{1}, \ldots, e_{d}\right\}$ be the standard orthonormal basis of $\mathbb{R}^{d}$. We write $\partial_{j}=\partial_{e_{j}}, \mathcal{D}_{j}=\mathcal{D}_{e_{j}}$. The Dunkl Laplacian $\Delta_{\kappa}$ is defined by

$$
\Delta_{\kappa}=\sum_{j=1}^{d} \mathcal{D}_{j}^{2}
$$

It has a following expression ([7]):

$$
\Delta_{\kappa} f(x)=\Delta f(x)+\sum_{\alpha \in R_{+}} \frac{2 k_{\alpha}}{\langle\alpha, x\rangle} \partial_{\alpha} f(x)-\sum_{\alpha \in R_{+}} \kappa_{\alpha} \frac{\langle\alpha, \alpha\rangle}{\langle\alpha, x\rangle^{2}}\left\{f(x)-f\left(r_{\alpha} x\right)\right\} .
$$

The Dunkl operators are homogeneous of degree -1 and the Dunkl Laplacian $\Delta_{\kappa}$ is homogeneous of degree -2 .

Write $\mathcal{D}=\left(\mathcal{D}_{1}, \ldots, \mathcal{D}_{d}\right)$. For $p \in \mathscr{P}$,

$$
p(\mathcal{D})=\sum_{g \in G} \mathcal{D}_{p}^{(g)} g
$$

where $\mathcal{D}_{p}^{(g)}(g \in G)$ are differential operators uniquely determined by $p$ and $\kappa$. Let $L_{p}$ denote the differential operators defined by

$$
L_{p}=\sum_{g \in G} \mathcal{D}_{p}^{(g)}\left(=\left.p(\mathcal{D})\right|_{G \text {-inv.poly. }}\right)
$$

For example, $L_{p}=\partial_{j}$ for $p(x)=x_{j}$ and

$$
L_{m_{2}}=\Delta+\sum_{\alpha \in R_{+}} \frac{2 k_{\alpha}}{\langle\alpha, x\rangle} \partial_{\alpha}\left(=\left.\Delta_{k}\right|_{G \text {-inv.poly. }}\right)
$$

for $m_{2}(x)=\|x\|^{2}=x_{1}^{2}+\cdots+x_{d}^{2}$. 
For any $y \in \mathbb{R}^{d}$, there exists a unique real analytic function $x \mapsto E_{\kappa}(x, y)=E(x, y)$ such that $\mathcal{D}_{j} E_{\kappa}(\cdot, y)=y_{j} E_{\kappa}(\cdot, y)(1 \leq j \leq d)$ and $E_{\kappa}(0, y)=1([16])$. We call $E_{\kappa}(x, y)$ the Dunkl kernel. If $\kappa=0, E_{0}(x, y)=\exp (\langle x, y\rangle)$.

Let $h_{\kappa}(x)$ denote the weight function defined by

$$
h_{\kappa}(x)=\prod_{\alpha \in R_{+}}|\langle\alpha, x\rangle|^{\kappa_{\alpha}} .
$$

The Dunkl transform of a function $f \in L^{1}\left(\mathbb{R}^{d}, h_{\kappa}^{2}(x) d x\right)$ is defined by

$$
\left(\mathscr{F}_{\kappa} f\right)(y)=b_{\kappa} \int_{\mathbb{R}^{d}} f(x) E_{\kappa}(x,-i y) h_{\kappa}(x) d x,
$$

where

$$
b_{\kappa}=\left(\int_{\mathbb{R}^{d}} h_{\kappa}^{2}(x) e^{-\|x\|^{2} / 2} d x\right)^{-1} .
$$

If $\kappa=0$, then $b_{0}=(2 \pi)^{-d / 2}$ and $\mathscr{F}_{0}$ is the Euclidean Fourier transform. The Dunkl transform has very nice properties as those of the Euclidean Fourier transform. For example,

$$
\mathscr{F}_{\kappa}\left(M_{x_{j}} f\right)=i \mathcal{D}_{j} \mathscr{F}_{\kappa} f \quad(1 \leq j \leq d),
$$

where $M_{p}$ denotes the multiplication operator by $p \in \mathscr{P}([9$, Corollary 7.7.1]) Moreover, the inversion formula and the Plancherel formula are known for the Dunkl transform ([16]).

\section{HOBSON'S FORMULA}

Now we state and prove an analogue of Hobson's formula for the Dunkl operators.

Theorem 1. If $p \in \mathscr{P}_{m}, f_{0} \in C^{\infty}((0, \infty))$, and $f(x)=f_{0}(\|x\|)$, then

$$
p(\mathcal{D}) f(x)=\sum_{j=0}^{[m / 2]} \frac{1}{2^{j} j !}\left[\left(\frac{1}{r} \frac{d}{d r}\right)^{m-j} f_{0}\right](\|x\|) \cdot \Delta_{\kappa}^{j} p(x) .
$$

Since $f(x)$ is $G$-invariant, the left hand side of (3.1) coincides with $L_{p} f(x)$. If $\kappa=0$, then Theorem 1 is the original Hobson's formula ([14], [15, p. 124]). If $G=\mathbb{Z}_{2}^{d}$, then Theorem 1 is given by Volkmer [30, Theorem 6].

Example 1. If $p(x)=\|x\|^{2}$, then $m=2$ and $p(\mathcal{D})=\Delta_{\kappa}$. By Theorem 1 we have

$$
\Delta_{\kappa} f(x)=L_{m_{2}} f(x)=f_{0}^{\prime \prime}(r)+\frac{d-1+2 \gamma_{k}}{r} f_{0}^{\prime}(r) \quad(r:=\|x\|) .
$$

If we put $f(x)=e^{-\|x\|^{2} / 2}$ in Theorem 1, then we have

$$
p(\mathcal{D}) e^{-\|x\|^{2} / 2}=\sum_{j=0}^{[m / 2]} \frac{(-1)^{m-j}}{2^{j} j !} e^{-\|x\|^{2} / 2} \Delta_{\kappa}^{j} p(x) .
$$

Proof of Theorem 1 First notice that the upper bound $[m / 2]$ in the summation in (3.1) may be replaced by $m$. We prove the theorem by induction on $m$. The case $m=0$ 
is trivial. Assume that (3.1) holds for any element of $\mathscr{P}_{m}$. Let $p \in \mathscr{P}_{m+1}$. Then $E p=(m+1) p$, where $E$ denotes the Euler operator

$$
E=\sum_{l=1}^{d} x_{l} \partial_{l}
$$

Thus

$$
p(\mathcal{D})=\frac{1}{m+1} \sum_{l=1}^{d} \mathcal{D}_{l}\left(\partial_{l} p\right)(\mathcal{D}) .
$$

Since $\partial_{l} p \in \mathscr{P}_{m}$, it follows from the induction hypothesis that

$$
p(\mathcal{D}) f(x)=\frac{1}{m+1} \sum_{l=1}^{d} \sum_{j=0}^{m} \frac{1}{2^{j} j !} \mathcal{D}_{l}\left\{\left[\left(\frac{1}{r} \frac{d}{d r}\right)^{m-j} f_{0}\right](\|x\|) \cdot \Delta_{\kappa}^{j} \partial_{l} p(x)\right\} .
$$

By the definition of the Dunkl operator $(2.2)$ and the chain rule, we have

$$
\begin{aligned}
\mathcal{D}_{l} & \left\{\left[\left(\frac{1}{r} \frac{d}{d r}\right)^{m-j} f_{0}\right](\|x\|) \cdot \Delta_{\kappa}^{j} \partial_{l} p(x)\right\} \\
& =\left[\left(\frac{1}{r} \frac{d}{d r}\right)^{m+1-j} f_{0}\right](\|x\|) \cdot x_{l} \Delta_{\kappa}^{j} \partial_{l} p(x)+\left[\left(\frac{1}{r} \frac{d}{d r}\right)^{m-j} f_{0}\right](\|x\|) \cdot \mathcal{D}_{l} \Delta_{\kappa}^{j} \partial_{l} p(x) \\
& =\left[\left(\frac{1}{r} \frac{d}{d r}\right)^{m+1-j} f_{0}\right](\|x\|) \cdot \Delta_{\kappa}^{j} x_{l} \partial_{l} p(x)-\left[\left(\frac{1}{r} \frac{d}{d r}\right)^{m+1-j} f_{0}\right](\|x\|) \cdot 2 j \mathcal{D}_{l} \Delta_{\kappa}^{j-1} \partial_{l} p(x) \\
& +\left[\left(\frac{1}{r} \frac{d}{d r}\right)^{m-j} f_{0}\right](\|x\|) \cdot \mathcal{D}_{l} \Delta_{\kappa}^{j} \partial_{l} p(x) .
\end{aligned}
$$

The last equality follows from

$$
\left[\Delta_{\kappa}^{j}, M_{x_{l}}\right]=2 j \mathcal{D}_{l} \Delta_{k}^{j-1},
$$

which is an easy consequence of $\left[\Delta_{\kappa}, M_{x_{l}}\right]=2 \mathcal{D}_{l}$ ([7, Proposition 2.2], [9, Lemma 7.1.9]). Substituting the above expression into (3.4), we have

$$
\begin{aligned}
p(\mathcal{D}) & =\sum_{j=0}^{m} \frac{1}{2^{j} j !}\left[\left(\frac{1}{r} \frac{d}{d r}\right)^{m+1-j} f_{0}\right](\|x\|) \cdot \Delta_{\kappa}^{j} p(x) \\
& -\sum_{l=1}^{d} \sum_{j=0}^{m} \frac{1}{2^{j-1}(j-1) !}\left[\left(\frac{1}{r} \frac{d}{d r}\right)^{m+1-j} f_{0}\right](\|x\|) \cdot \mathcal{D}_{l} \Delta_{\kappa}^{j-1} \partial_{l} p(x) \\
& +\sum_{l=1}^{d} \sum_{j=0}^{m} \frac{1}{2^{j} j !}\left[\left(\frac{1}{r} \frac{d}{d r}\right)^{m-j} f_{0}\right](\|x\|) \cdot \mathcal{D}_{l} \Delta_{\kappa}^{j} \partial_{l} p(x) \\
& =\sum_{j=0}^{m+1} \frac{1}{2^{j} j !}\left[\left(\frac{1}{r} \frac{d}{d r}\right)^{m+1-j} f_{0}\right](\|x\|) \cdot \Delta_{\kappa}^{j} p(x),
\end{aligned}
$$

by using $E p=(m+1) p$ and changing a summation index. Hence the Theorem is proved. 
Remark 1. We imitate the proof of Hobson's formula for $\kappa=0$ given by Nomura 20] in the proof of Theorem 1 1 . Use of the Euler operator and induction on the degree of $p$ also work well in our case. In the case of $\kappa=0$, Nomura proceeds after (3.4) by using $\left[\partial_{l}, \Delta^{j}\right]=0$ and $\left[\Delta^{j}, E\right]=2 j \Delta^{j}$. Instead of doing in the similar way, we use 3.5 , because for general $\kappa$ we do not have a nice formula for $\left[\partial_{l}, \Delta_{\kappa}^{j}\right]$.

Remark 2. Theorem 1 is closely related with the formula ([13, Proposition 3.4])

$$
p(\mathcal{D})=\frac{1}{m !}\left(\text { ad } \frac{\Delta_{k}}{2}\right)^{m} M_{p} \quad\left(p \in \mathscr{P}_{m}\right),
$$

which can be proved by using $\left[\Delta_{\kappa}, M_{x_{l}}\right]=2 \mathcal{D}_{l}$ (see [17, Section 3]).

\section{Applications of Hobson's formula}

In this section, we give some applications of Hobson's formula (Theorem 1). Though they are all known results, Hobson's formula provides simpler alternative proofs.

4.1. $\kappa$-harmonic polynomials. A polynomial $p$ is called $\kappa$-harmonic if $\Delta_{\kappa} p=0$. Let $\mathscr{H}_{m, \kappa}$ denote the space of $\kappa$-harmonic polynomials in $\mathscr{P}_{m}$.

An explicit formula for the projection operator from $\mathscr{P}_{m}$ to $\mathscr{H}_{m, \kappa}$ is derived from Hobson's formula.

It follows from $(3.2)$ by putting $f(x)=\|x\|^{s}$ that

$$
\Delta_{\kappa}\|x\|^{s}=s\left(s+2 \lambda_{\kappa}\right)\|x\|^{s-2} .
$$

In particular, $\Delta_{\kappa}\|x\|^{-2 \lambda_{\kappa}}=0$.

Applying Theorem 1 to $f(x)=\|x\|^{-2 \lambda_{\kappa}}$, we have

$$
p(\mathcal{D})\left(\|x\|^{-2 \lambda_{\kappa}}\right)=\sum_{j=0}^{[m / 2]} \frac{(-1)^{m-j} 2^{m}\left(\lambda_{\kappa}\right)_{m-j}}{2^{2 j} j !}\|x\|^{-2\left(\lambda_{\kappa}+m-j\right)} \Delta_{\kappa}^{j} p(x) .
$$

Thus we have

$$
p(x)=\|x\|^{2 \lambda_{\kappa}+2 m} p(\mathcal{D})\left(\|x\|^{-2 \lambda_{\kappa}}\right)-\sum_{j=1}^{[m / 2]} \frac{1}{2^{2 j} j !\left(-\lambda_{\kappa}-m+1\right)_{j}}\|x\|^{2 j} \Delta_{\kappa}^{j} p(x) .
$$

The first term $\|x\|^{2 \lambda_{\kappa}+2 m} p(\mathcal{D})\left(\|x\|^{-2 \lambda_{\kappa}}\right)$ of the right hand side of 4.2 is $\kappa$-harmonic by [31, Theorem 2.3] and the remaining term is divisible by $\|x\|^{2}$. Define the Clebsch projection $\operatorname{proj}_{m, \kappa}$ by

$$
\operatorname{proj}_{m, \kappa} p(x)=\|x\|^{2 \lambda_{\kappa}+2 m} p(\mathcal{D})\left(\|x\|^{-2 \lambda_{\kappa}}\right) \quad\left(p \in \mathscr{P}_{m}\right) .
$$

Then by 4.1) we have

$$
\operatorname{proj}_{m, \kappa} p(x)=\sum_{j=0}^{[m / 2]} \frac{1}{2^{2 j} j !\left(-\lambda_{\kappa}-m+1\right)_{j}}\|x\|^{2 j} \Delta_{\kappa}^{j} p(x) .
$$

The formula (4.4) is proved by Dunkl ([6], [9, Theorem 7.1.15]) using a different method. 
If $p \in \mathscr{H}_{m, \kappa}$, then $\operatorname{proj}_{m, \kappa} p(x)=p(x)$. Hence the mapping $\operatorname{proj}_{m, \kappa}: \mathscr{P}_{m} \rightarrow \mathscr{H}_{m, \kappa}$ is surjective, which gives Maxwell's representation formula for $\kappa$-harmonic functions. The Clebsch projection $\operatorname{proj}_{m, \kappa}$ is not injective. Choices of a subset of $\mathscr{P}_{\kappa}$ whose image under $\operatorname{proj}_{m, \kappa}$ gives a basis of $\mathscr{H}_{m, \kappa}$ are studied by Xu [31]. If $\kappa=0$, formulae for $\operatorname{proj}_{m, 0}$ and its surjectivity are classical well-known facts (see [15], [5, Vol. I, Ch. VII, §4, 5], [19, Ch $1, \S 6]$, [28, Ch. IV $\S 2.5])$.

4.2. Bochner-Hecke identity. As in the Euclidean case ([25, 26]), an analogue of the Bochner-Hecke identity for the Dunkl transform follows from Hobson's formula (Theorem 1).

Let $d \omega$ denote the surface measure on $S^{d-1}$ induced from the measure $d x$ on $\mathbb{R}^{d}$. Define

$$
\sigma_{d-1, \kappa}=\int_{S^{d-1}} h_{\kappa}(x) d \omega(x) .
$$

Using polar coordinates we have

$$
b_{\kappa}^{-1}=2^{\lambda_{\kappa}} \sigma_{d-1, \kappa} \Gamma\left(\lambda_{\kappa}+1\right) .
$$

By [8, Proposition 2.8] (see also [29, Theorem 1.4.2]), we have

$$
\int_{S^{d-1}} E_{\kappa}(x,-i y) h_{\kappa}^{2}(x) d \omega(x)=\sigma_{d-1, \kappa} \Gamma\left(\lambda_{\kappa}+1\right)\left(\frac{\|y\|}{2}\right)^{-\lambda_{\kappa}} J_{\lambda_{\kappa}}(\|y\|) .
$$

Here $J_{\nu}(r)$ is the Bessel function of the first kind

$$
J_{\nu}(r)=\sum_{j=0}^{\infty} \frac{(-1)^{j}(r / 2)^{\nu+2 j}}{j ! \Gamma(\nu+j+1)}
$$

The following proposition is proved by González Vieli [12, Lemma 3.1]. We give a simple proof by using Hobson's formula (Theorem 1).

Proposition 2. For $p \in \mathscr{P}_{m}$, we have

$$
b_{\kappa} \int_{S^{d-1}} p(x) E_{\kappa}(x,-i y) h_{\kappa}^{2}(x) d \omega(x)=(-i)^{m} \sum_{j=0}^{[m / 2]} \frac{(-1)^{j}}{2^{j} j !} \frac{J_{\lambda_{\kappa}+m-j}(\|y\|)}{\|y\|^{\lambda_{\kappa}+m-j}} \Delta_{\kappa}^{j} p(y) .
$$

In particular, if $p \in \mathscr{H}_{m, \kappa}$, then

$$
b_{\kappa} \int_{S^{d-1}} p(x) E_{\kappa}(x,-i y) h_{\kappa}^{2}(x) d \omega(x)=\frac{J_{\lambda_{\kappa}+m}(\|y\|)}{\|y\|^{\lambda_{\kappa}+m}} p(-i y) .
$$

Proof. By applying $p(\mathcal{D})$ with the variable $y$ to 4.7$)$ and using $\mathcal{D}_{j} E_{\kappa}(x,-i y)=-i x_{j} E_{\kappa}(x,-i y)$, Theorem 1 and a formula for the Bessel function [10, Ch VII, (51)]

the proposition follows.

$$
\frac{1}{r} \frac{d}{d r}\left(r^{-\nu} J_{\nu}(r)\right)=-r^{-\nu-1} J_{\nu+1}(r)
$$

The formula (4.9) is given by [27, Theorem 3.1]. For $\kappa=0,4.9$ ) is given by [4, Lemma 2.6.2], [1, Lemma 9.10.2].

We have the following Theorem ([12, Theorem 4.1]) as a corollary of Proposition 2. 
Theorem 3. For $p \in \mathscr{P}_{m}$ and a radial function $f(x)=f_{0}(\|x\|)$ we have

$$
\mathscr{F}_{\kappa}\left(M_{p} f\right)(y)=(-i)^{m} \sum_{j=0}^{[m / 2]} \frac{(-1)^{j}}{2^{j} j !}\left(\mathscr{H}_{\lambda_{\kappa}+m-j} f_{0}\right)(\|y\|) \Delta_{\kappa}^{j} p(y),
$$

where

$$
\left(\mathscr{H}_{\nu} f\right)(s)=\int_{0}^{\infty} f(r) \frac{J_{\nu}(r s)}{(r s)^{\nu}} r^{2 \nu+1} d r
$$

denote the Hankel transform. In particular, if $p \in \mathscr{H}_{m, \kappa}$, then

$$
\mathscr{F}_{\kappa}\left(M_{p} f\right)(y)=(-i)^{m}\left(\mathscr{H}_{\lambda_{\kappa}+m} f_{0}\right)(\|y\|) p(y) .
$$

If $\kappa=0$, Theorem 3 is given by [26, Theorem 1]. If $\kappa=0,44.10]$ is given by 3 , Theorem 2], [4, Theorem 2.6.1]. For general $\kappa,(4.10)$ is given by [2, Theorem 3.15] and [27, Theorem 3.2].

Since $\mathscr{H}_{\lambda_{\kappa}+m} f_{0}=f_{0}$ for $f_{0}(r)=e^{-r^{2} / 2}([11,8.6(10)])$, we have an analogue of the Bochner-Hecke identity from Theorem 3 .

Corollary 4. For $p \in \mathscr{P}_{m}$ we have

$$
b_{\kappa} \int_{\mathbb{R}^{d}} p(x) e^{-\|x\|^{2} / 2} h_{\kappa}^{2}(x) d x=(-i)^{m} e^{-\|y\|^{2} / 2} \sum_{j=0}^{[m / 2]} \frac{(-1)^{j}}{2^{j} j !} \Delta_{\kappa}^{j} p(y) .
$$

In particular, if $p \in \mathscr{H}_{m, \kappa}$, then

$$
b_{\kappa} \int_{\mathbb{R}^{d}} p(x) e^{-\|x\|^{2} / 2} h_{\kappa}^{2}(x) d x=(-i)^{m} p(y) e^{-\|y\|^{2} / 2} .
$$

If $\kappa=0$, then (4.11) is the Bochner-Hecke identity (cf. [4, Theorem 2.6.3], 24, Theorem 3.10], [1, Theorem 9.10.3]). (4.11) for general $\kappa$ is given by [2, Example 3.16].

We have the following corollary from $(3.3)$ and Corollary 4

Corollary 5. The following conditions (1) (3) for $p \in \mathscr{P}_{m}$ are mutually equivalent.

(1) $p \in \mathscr{H}_{m, \kappa}$

(2) $b_{\kappa} \int_{\mathbb{R}^{d}} p(x) e^{-\|x\|^{2} / 2} h_{\kappa}^{2}(x) d x=(-i)^{m} p(y) e^{-\|y\|^{2} / 2}$

(3) $p(\mathcal{D}) e^{-\|x\|^{2} / 2}=p(-x) e^{-\|x\|^{2} / 2}$

4.3. Pizzetti's formula. We have Pizzetti's formula for the Dunkl analysis as a corollary of Proposition 2 .

Corollary 6. For $p \in \mathscr{P}$ we have

$$
b_{\kappa} \int_{S^{d-1}} p(x) h_{\kappa}^{2}(x) d \omega(x)=\sum_{l=0}^{\infty} \frac{(-1)^{l} 2^{-\lambda_{\kappa}}}{2^{2 l} l ! \Gamma\left(\lambda_{\kappa}+l+1\right)}\left(\Delta_{\kappa}^{l} p\right)(0) .
$$

Proof. First assume that $p \in \mathscr{P}_{m}$. Substituting $y=0$ to the formula in Proposition 2 . we have

$$
b_{\kappa} \int_{S^{d-1}} p(x) h_{\kappa}^{2}(x) d \omega(x)=(-i)^{m} \sum_{j=0}^{[m / 2]} \frac{(-1)^{j}}{2^{j} j !} \frac{2^{-\lambda_{\kappa}-m+j}}{\Gamma\left(\lambda_{\kappa}+m-j+1\right)}\left(\Delta_{\kappa}^{j} p\right)(0) .
$$


Notice that $\left(\Delta_{\kappa}^{j} p\right)(0)=0$ for any $j \in \mathbb{Z}_{+}$, if $m$ is odd. Therefore the right hand side of (4.13) is zero, if $m$ is odd. If $m$ is even, say $m=2 l$, then 4.13 becomes

$$
b_{\kappa} \int_{S^{d-1}} p(x) h_{\kappa}^{2}(x) d \omega(x)=\frac{(-1)^{l} 2^{-\lambda_{\kappa}}}{2^{2 l} l ! \Gamma\left(\lambda_{\kappa}+l+1\right)}\left(\Delta_{\kappa}^{l} p\right)(0) .
$$

The formula 4.12 for general $p \in \mathscr{P}$ follows by applying above results for each homogeneous component and summing up.

Corollary 6 is a special case of [18, Theorem 4.17]. If $\kappa=0$, Corollary 6 is a classical formula of Pizzetti ([21], [5, Vol II, Ch. IV, §3.4].

If $p \in \mathscr{H}_{m, \kappa}, 4.12$ becomes the mean value property

$$
\sigma_{d-1, \kappa}^{-1} \int_{S^{d-1}} p(x) h_{\kappa}^{2}(x) d \omega(x)=p(0) .
$$

4.4. Hermite polynomials. For $p \in \mathscr{P}_{m}$, define the Hermite polynomial $H_{p, \kappa}(x)$ by

$$
H_{p, \kappa}(x)=\left(-\frac{1}{2}\right)^{m} e^{\|x\|^{2}} p(\mathcal{D}) e^{-\|x\|^{2}} .
$$

By Hobson's formula (Theorem 1) we have

$$
H_{p, \kappa}(x)=\sum_{j=0}^{[m / 2]} \frac{(-1)^{j}}{2^{2 j} j !} \Delta_{\kappa}^{j} p(x) .
$$

In particular, if $p \in \mathscr{H}_{m, \kappa}$, then $H_{p, \kappa}=p$.

The Dunkl transform of the Hermite function $h_{p, \kappa}(x):=e^{-\|x\|^{2} / 2} H_{p, \kappa}(x)$ is given by

$$
\mathscr{F}_{\kappa} h_{p, \kappa}=(-i)^{m} h_{p, \kappa} \text {. }
$$

González Vieli [12, §5] proves 4.16) by using (4.15) and Corollary 4.

The above results for the Hermite polynomials are first given by Rösler [22] (with different proof). If $\kappa=0$, above formulae are proved by Strasburger [25, 26] by using Hobson's formula.

\section{ACKNOWLEDGEMENT}

The author thanks Professor Takaaki Nomura and Professor Hiroshi Oda for helpful discussions.

\section{REFERENCES}

[1] G.E. Andrews, R. Askey, R. Roy, Special Functions, Cambridge University Press, 1999.

[2] S. Bent Saïd, On the integrability of a representation of $\mathfrak{s l}(2, \mathbb{R})$, Jour. Funct. Anal. 250 (2007), 249-264.

[3] S. Bochner, Theta relations with spherical harmonics, Proc. Nat. Acad. Sci. U.S.A. 37 (1951), 804808.

[4] S. Bochner, Harmonic Analysis and the Theory of Probability, University of California Press, 1955.

[5] R. Courant and D. Hilbert, Methoden der mathematischen Physik I, II, Springer, 1924, 1937.

[6] C.F. Dunkl, Reflection groups and orthogonal polynomials on the sphere, Math. Z. 197 (1988), 33-60. 
[7] C.F. Dunkl, Differential-difference operators associated to reflection groups, Trans. Amer. Math. Soc. 311 (1989), 167-183.

[8] C.F. Dunkl, Hankel transforms associated to finite reflection groups, Contemporary Mathematics 138 (1992), 123-138.

[9] C.F. Dunkl and Y. Xu, Orthogonal Polynomials of Several Variables Second. Ed., Cambridge University Press, 2014.

[10] A. Erdélyi etl al., Higher Transcendental Functions II, McGraw-Hill, 1955.

[11] A. Erdélyi et al., Tables of Integral Transforms II, McGraw-Hill, 1954.

[12] F.J. González Vieli, Generalization of the Bochner identity for the Dunkl transform, Integral Transforms Spec. Funct. 25 (2014), 930-936.

[13] G.J. Heckman, A remark on the Dunkl differential-difference operators, In Harmonic Analysis on Reductive Groups. Progress in Math. 101, Birkhäuser (1991), 181-191.

[14] E.W. Hobson, On a theorem in the differential calculus, Messenger Math. 23 (1894), 115-119.

[15] E.W. Hobson, The Theory of Spherical and Ellipsoidal Harmonics, 2nd ed., Cambridge University Press, 1955.

[16] M.F.E. de Jeu, The Dunkl transform, Invent. Math. 113 (1993), 147-162.

[17] M.F.E. de Jeu, Paley-Wiener theorems for the Dunkl transform, Trans. Amer. Math. Soc. 358 (2006), $4225-4250$.

[18] H. Mejjaoli and K. Trimèche, Mean value property associated with the Dunkl Laplacian, Integral Transform. Spec. Funct. 12 (2001), 279-302.

[19] C, Müller, Analysis of Spherical Symmetries in Euclidean Spaces, Springer Verlag, 1998.

[20] T. Nomura, A proof of Hobson's formula with the Euler operator, to appear in Kyushu J. of Math.

[21] P. Pizzetti, Sulla media dei valori che una funzione dei punti dello spazio assume alla superficie di una sfera, Rend. Lincei, 5 (1909), 182-185.

[22] M. Rösler, Generalized Hermite polynomials and the heat equation for Dunkl operators, Commun. Math. Phys. 192 (1998), 519-541.

[23] M. Rösler, Dunkl Operators: Theory and Applications, Orthogonal Polynomials and Special Functions, LNM 1817 (2003), 93-135.

[24] E.M. Stein and G. Weiss, Introduction to Fourier Analysis on Euclidean Spaces, Princeton University Press, 1971.

[25] A. Strasburger, Differential operators of gradient type associated with spherical harmonics, Ann. Polon. Math. 53, (1991), 161-183.

[26] A. Strasburger, A generalization of the Bochner identity, Expo. Math. 11 (1993), 153-157.

[27] K. Trimèche, Generalized Bochner-Hecke theorems and application to homogeneous distributions in Dunkl's theory, Comm. Math. Anal. 3 (2007), 54-68.

[28] N.Ja. Vilenkin, Special Functions and the Theory of Group Representations, American Mathematical Society, 1968.

[29] N.Ja. Vilenkin and A.U. Klimyk, Representation of Lie Groups and Special Functions: Recent Advances, Kluwer, 1995.

[30] H. Volkmer, Generalized ellipsoidal and sphero-conal harmonics, SIGMA 2 (2006), 071, 16 pages.

[31] Y. Xu, Harmonic polynomials associated with reflection groups, Canad. Math. Bull. 43 (2000), 496507.

School of Science and Technology, Kwansei Gakuin University, 2-1 Gakuen, Sanda, HYOGO 669-1337, JAPAN

E-mail address: shimeno@kwansei.ac.jp 